\title{
Design for Reactive Power Compensator Based on Buck-Boost AC/AC Chopper
}

\author{
MA Tianshun ${ }^{1, a}$, MA Lixin ${ }^{2, b}$ and Liu Yukuan ${ }^{3, c}$ \\ ${ }^{1,2,3}$ Department of Electrical Engineering School of Optical-Electrical and Computer Engineering, \\ University of Shanghai for Science \&Technology,Shanghai,200093,China \\ amatianshun308@sina.com, ${ }^{\mathrm{b}}$ malxaii@163.com, cyukuanliu@126.com
}

Keywords: buck-boost circuit; reactive power compensation; direct current control; Matlab

\begin{abstract}
Aiming at the problem of voltage reduction and voltage distortion due to the extensive use of inductive loads, and some shortcomings of existing reactive power compensation devices, a new AC-AC chopper based on compensator is proposed. The basic principles, compensation characteristics and topology of the Buck-Boost AC-AC chopper based var compensator are described, the mathematical model of Buck-Boost based on state-space equation is established, the relationship between duty ratio and compensation current is derived. The instantaneous reactive power theory and direct current control method are adopted to build a MATLAB model. The results indicate that the real-time compensation of reactive power in grid side can be realized by this var compensator based on a direct controlled Buck-Boost AC-AC chopper, it has potential application value.
\end{abstract}

\section{Introduction}

With the rapid development of industrial processes, power grid demands change drastically, especially the growing of impact load (such as a large rolling mill, electric tram, power converter device). Generally speaking, the booting process of those electrical equipment is short, during the booting process frequency keep a high level, large amounts of dynamic reactive power was absorbed frequently, those factor cause rapid bus voltage fluctuation, bringing power grid stability a negative impact. It is urgent to resolve the problem such as how to improve the power factor of the power system, how to improve voltage stability of user and the grid, how to balanced three-phase active and reactive loads have become a serious problem [1].

\section{The basic principles of the Buck-Boost AC-AC chopper based var compensator}

Buck-Boost AC-AC chopper based var compensator consists of Filter in grid side, AC/AC Chopper and Compensation capacitor. Filter is mainly used to eliminate the interference of multiple frequency harmonics and edge frequency harmonic component of breaker to power grid.

If a Buck-Boost AC-AC chopper based var compensator is added between grid and capacitor, by controlling switch status of chopper circuit, we can get a capacitive current whose angle 90 degrees ahead of the grid voltage [2]. The amplitude of the current is adjustable. This capacitive current can continuously and dynamically compensate reactive power of $\mathrm{AC}$ side, and finally make the power factor of grid is 1 .

The Figure1shows the reactive power compensator principle diagram based on Buck-boost AC/AC chopper, Q1, Q2 and Q3, Q4 are two bidirectional switch respectively consisting of two IGBT series reversely [3]. These two switches complementary closing. Inductor $L_{f}$ is used to storage. Capacitor $C_{f}$ is used to eliminate the higher harmonic components of switching frequency and its multiples frequency. $\mathrm{C}$ is reactive power compensation capacitor. The two switchs, $\mathrm{s} 1$ and $\mathrm{s} 2$, works as complementary roles. For purposes of analysis, Figure 2(a) equivalent circuit can be simplified as shown in Figure 2(b). 


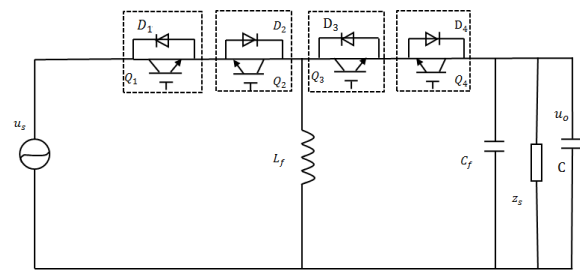

(a)Circuit topology

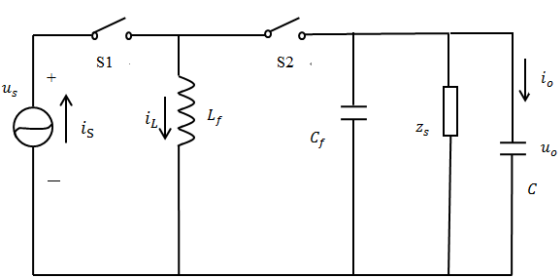

(b)Equivalent circuit

Fig.1 Reactive power compensator principle diagram based on Buck-boost AC/AC chopper

Under the conditions of all the components in circuit is ideal devices. The input voltage is expressed as $u_{s}$, dealing with the chopper circuit, then obtained a voltage expressed as $u_{0}$.Switching frequency is $f_{s}$.Switching cycle $T_{s}=1 / f_{s}$. Duty-cycle of S1 is $\mathrm{D}(0<\mathrm{D}<1)$. Define a functions $\xi(t) 、 \delta(t)$.When S1 is turned on, S2 turned off, $\xi(t)=1, \delta(t)=0$, When S2 is turned on, S1 turned off, $\xi(t)=0 、 \delta(t)=1_{\text {[4].That is: }}$

$$
\begin{gathered}
\xi(t)=\left\{\begin{array}{c}
1\left(0 \leq t \leq D T_{s}\right) \\
0\left(D T_{s} \leq t \leq T_{s}\right)
\end{array}\right. \\
\delta(t)=\left\{\begin{array}{l}
0\left(0 \leq t \leq D T_{s}\right) \\
1\left(D T_{s} \leq t \leq T_{s}\right)
\end{array}\right.
\end{gathered}
$$

In order to facilitate analysis, consider voltage at the end of the inductor. When the high-frequency switch S1 is turned on, S2 turned off, voltage of inductor $u_{L}=u_{s}$. When $\mathrm{S} 1$ is turned off, $\mathrm{S} 2$ turned on, $u_{L}=u_{0}$. That is:

$$
\begin{gathered}
0 \leq t \leq \mathrm{D} T_{s} \text { 时, } u_{L}=u_{s} ; \\
\mathrm{D} T_{s} \leq t \leq T_{s} \text { 时 } u_{L}=u_{0}
\end{gathered}
$$

So, that: $u_{L}=\xi(\mathrm{t}) u_{s}+\delta(t) u_{o}$

According the formula to Fourier series expansion:

$$
\begin{gathered}
\xi(t)=D+\frac{2}{\pi} \sum_{K=1}^{\infty} \frac{1}{K} \sin \Phi_{K}\left\lceil\cos \left(k \omega_{0}-\Phi_{K}\right)\right. \\
\delta(t)=1-D+\frac{2}{\pi} \sum_{K=1}^{\infty} \frac{1}{K} \sin \Phi_{K} \cdot \cos \left(\mathrm{k} \omega_{0}-\Phi_{K}\right)
\end{gathered}
$$

Fundamental frequency of $\xi(t) 、 \delta(t)$ is $\omega_{0}, \omega_{0}=2 \pi / T_{s}$. The initial phase angle $\Phi_{K}=k \pi D$. Because the average value of $u_{L}$ is $0, \xi(\mathrm{t}) u_{s}+\delta(t) u_{o}=0$.If $u_{s}=U_{s} \sin \omega_{s}$ :

$$
u_{o}=\frac{\mathrm{D}+\frac{2}{\pi} \sum_{K=1}^{\infty} \frac{1}{K} \sin \Phi_{K} \cdot \cos \left(\mathrm{k} \omega_{0}-\Phi_{K}\right)}{-\left[1-\mathrm{D}+\frac{2}{\pi} \sum_{K=1}^{\infty} \frac{1}{K} \sin \Phi_{K} \cdot \cos \left(\mathrm{k} \omega_{0}-\Phi_{K}\right) U_{s} \sin \omega_{s}\right.}
$$

After ignoring latter part of $\delta(\mathrm{t})$, the formula changed:

$$
\begin{aligned}
u_{o}=- & \frac{D}{1-\mathrm{D}} U_{s} \sin \omega_{s}-\frac{2 U_{s}}{(1-\mathrm{D}) \pi} \sum_{K=1}^{\infty} \frac{1}{K}-\sin \Phi_{K} * \\
& \left\{\sin \left[\left(k \omega_{0}+\omega_{s}\right) t-\Phi_{K}\right]-\sin \left[\left(k \omega_{0}-\omega_{s}\right) t-\Phi_{K}\right]\right\}
\end{aligned}
$$

Analysis of the formula (6), in addition to containing the fundamental frequency components, there are higher harmonic components, high-frequency part can be filted out by the low-pass filter, so get the following equation:

$$
u_{o}=-\frac{D}{1-\mathrm{D}} U_{s} \sin \omega_{\mathrm{s}}=-\frac{D}{1-\mathrm{D}} u_{s}
$$




$$
i_{o}=\frac{u_{o}}{-j \frac{1}{\omega_{s} c}}=j \omega c u_{o}=-\frac{\omega_{s} C D}{(1-\mathrm{D})} U_{s} \cos \omega_{s} t
$$

Formula (8) showed that, Buck-Boost AC-AC chopper based var compensator can be viewed as a capacitor whcih capacitance value can be adjusted continuous. We can complete reactive power dynamic compensation of the grid by controlling the duty-cycle of the switch.[5].

\section{Simulation and experimental verification}

To verify the rationality and right of reactive power compensation featureand direct current control strategy mentioned in this paper, we use the Simulink component library of MATLAB to build the simulation model of Buck-Boost AC-AC chopper based var compensator whose line voltage is $220 \mathrm{~V} / 50 \mathrm{~Hz}$. Reactive power of load $\mathrm{Q}=3 \mathrm{kvar}$; Switching frequency $=10 \mathrm{kHz}$; Inductor $=10 \mathrm{H}$; Filter capacitor $=20 \mathrm{uF}$; Compensation capacitor $\mathrm{C}=2 \mathrm{mF}$.

Figure 2(a),(b),(c) shows the compensation current waveform of grid getting from the output voltage and Buck-Boost AC-AC chopper based var compensator when duty-cycle is 0.2, 0.5, 0.8. Simulation result shows that compensation current is the capacitive current whose frequency is the same as output voltage and ahead of phase angle of output voltage, which can compensate grid reactive power.From the experimental results, we know that we can control the size of compensation current by regulating the duty-cycle of Buck-Boost AC-AC chopper based var compensator. As the picture shows, when duty-cycle changes from 0.2 to 0.8 , compensation current changes from 34.4A to 550A at the same time, which shows that Buck-Boost AC-AC chopper based var compensator has a bigger adjusting range. Figure 4(d), (e) shows the waveform of grid voltage and current before and after using the Var compensator. From the simulation result, we can know that before using the Var compensator, Power factor angle of system $\alpha=52^{\circ}$,Power factor $\lambda=0.62$.After using the Buck-Boost AC-AC chopper based var compensator, Power factor angle $\alpha=9^{\circ}$. Grid voltage and current in frequency and phase is almost synchronization and the power factor is approximately 1 , which verifies the veracity and feasibility of control strategy and the effectiveness of Buck-Boost AC-AC chopper based var compensator.
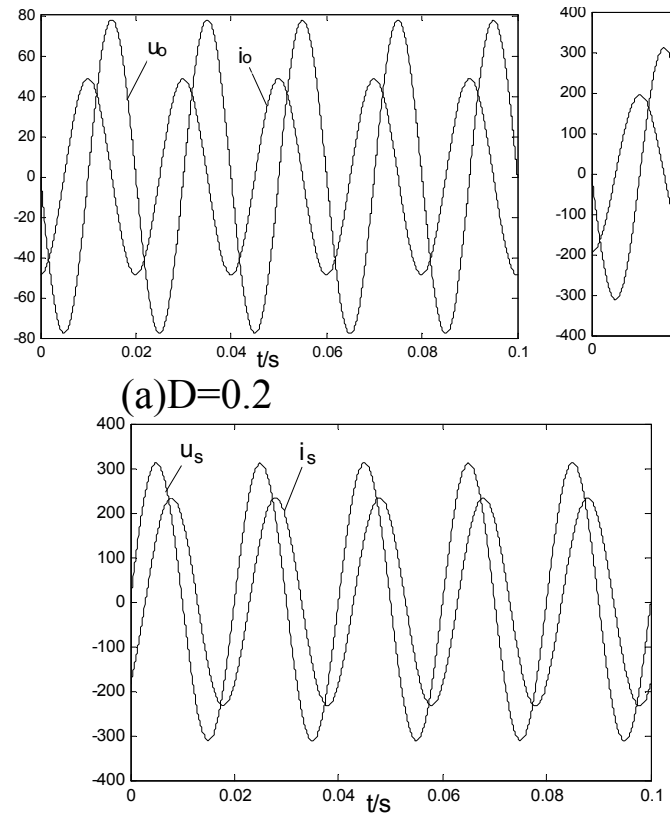

(d)voltage and current before compensation

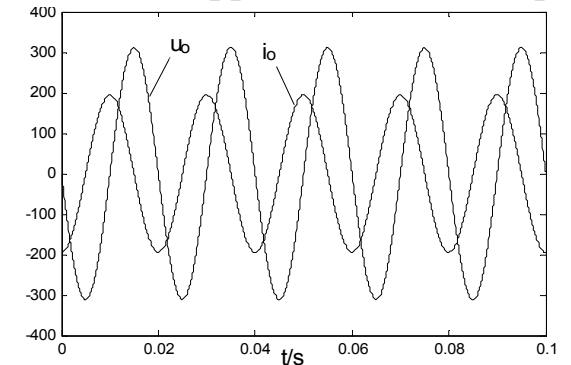

(b) $\mathrm{D}=0.5$

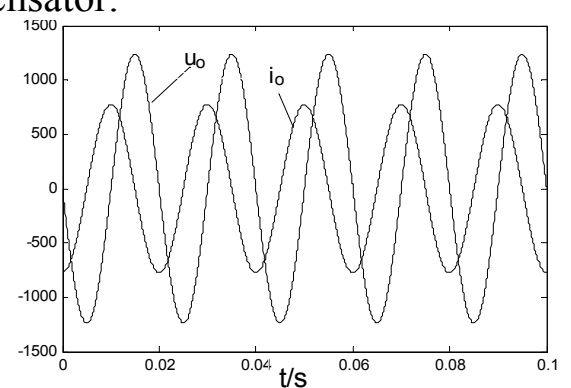

(c) $\mathrm{D}=0.8$

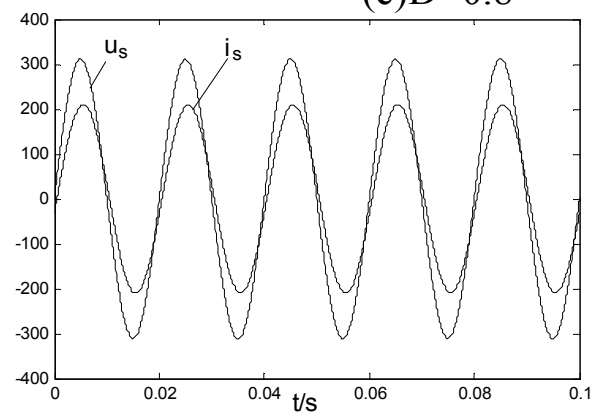

(e)voltage and current after compensation

Fig. 2 The results of simulation diagram

\section{Conclusion}

According to the analysis of the basic principles and topology of the Buck-Boost AC-AC 
chopper based var compensator, we get the relationship between compensation current and duty-cycle. Using PWM technology to control the compensator and simulation results of MATLAB software show that: Buck-Boost AC-AC chopper based var compensator can real-time and tracking compensating to reactive power frequency.

\section{References}

[1] Ding Mingchang, Zhang Youjun, Ren Yongbao.Research On Cascaded Buck- boost AC/AC Converter [J]. Electric Power Automation Equiment, 2010,30(9):46-50.

[2] Zhang Fei,Xu Jianping,Yang Ping,Chen Zhangyong. Two-switch Pseudo Continuous Conduction Mode Buck-Boost Power Factor Correction Converter [J]. Proceeding of the CSEE, 2012, 32(9):56-61.

[3] Chen Jiawei, Chen Jie, Gong Chunying. A Novel Dual Boost/Dual Buck AC-AC Converter[C]// Proceedings of IEEE 6th International Conference on Power Electronics and Motin Control Conference (IPEMC). Wuhan, China: IEEE, 2009: 1775-1779.

[4] Hamid R.Karshenas, Javad.Mousavi. A New Direct Sinusoidal Input/Output ac-ac Converter with Unidirectional Switches[C]// Proceedings of International Conference on Electrical Machines and Systems (ICEMS). Wuhan, China: IEEE, 2008: 1885-1890.

[5] Ashwin Kumar Sahoo, J.Meenakshi, S.S.Dash, T.Thyagaraian. Analysis and simulation of matrix converter using PSIM [C]// Proceedings of 7th International Conference on Power Electronics (ICPE). IEEE, 2007:414-419. 